\title{
ON ABSOLUTE CESÁRO SUMMABLITY OF FOURIER SERIES FOR ALMOST PERIODIC FUNCTIONS WITH LIMITING POINTS AT ZERO
}

\author{
YU.KH. KHASANOV
}

\begin{abstract}
In the paper we establish some tests for absolute Cesáro summability of the Fourier series for almost periodic functions in the Besicovitch space. We consider the case, when the Fourier exponents have a limiting point at zero and as a structure characteristics of the studied function, we use a high order averaging modulus.
\end{abstract}

Keywords: absolute summability, almost periodic function, Fourier series, Fourier exponents, limiting point at zero, averaging module.

Mathematics Subject Classification: 42A24, 42A75

\section{INTRODUCTION}

The scalar series

$$
\sum_{n=0}^{\infty} a_{n}
$$

is called absolute summable by Cesáro method of order $\alpha,(-1<\alpha<\infty)$ or $|C, \alpha|$-summable if

where

$$
\sum_{n=1}^{\infty}\left|\sigma_{n}^{\alpha}-\sigma_{n-1}^{\alpha}\right|<\infty
$$

$$
\sigma_{n}^{\alpha}=\sum_{k=0}^{\infty}\left(A_{n}^{\alpha}\right)^{-1} A_{n-k}^{\alpha} a_{k}(n=1,2, \cdots), \quad A_{n}^{\alpha}=\frac{(\alpha+1)(\alpha+2) \cdots(\alpha+n)}{n !} .
$$

The works devoted to the absolute Cesáro summability of arbitrary orthogonal series and, in particular, of Fourier series, appeared first in the beginning of 60s in the previous century and belonged mostly to Soviet and Hungarian mathematicians [1]-[6]. The main results of these studies can be found in works [5] and [7].

By the trigonometric system of a $2 \pi$-periodic function $f(x) \in L_{2}$ with the Fourier series

$$
\sum_{n=0}^{\infty}\left(a_{n} \cos n x+b_{n} \sin n x\right)
$$

L. Leindler [1] established some sufficient conditions for $|C, \alpha|$-summability of Fourier series for various values $\alpha>-1$. In the same work there were established similar results for the series over arbitrary orthonormal systems of functions $\{\varphi(x)\}$ defined on a finite interval $[a, b]$. A particular case of the Leindler's results corresponding to $|C, 1|$-summability almost everywhere of the Fourier series was established in work by K. Tandori [2].

Yu.Kh. Khasanov, On absolute Cesáro summablity of Fourier series for Almost Periodic FUNCTIONS WITH LIMITING POINTS AT ZERO.

(C) Khasanov YU.KH. 2016.

Submitted October 16, 2015. 
Analogues of the Leindler's results for functions $f(x) \in L_{p},(1<p \leqslant 2)$ in general constructive or structural terms were considered in works by M.F. Timan [3] and L.V. Grepachevskaya [5]. The authors established that in the case of monotonous Fourier coefficients, the considered conditions are also necessary. As $\alpha=0, p=2$, the results by L.V. Grepachevskaya [5] were obtained by S.B. Stechkin [4]. We also mention that L.V. Grepachevskaya 5] established the necessity of $|C, \alpha|$-summability of Fourier series over Rademacher system in the case of monotonous coefficients.

In the present work we provide some sufficient conditions of absolute Cesáro summability of Fourier series for almost periodic in the Besicovitch sense functions.

By $B_{p},(1 \leqslant p<\infty)$, we denote the space of almost periodic Besicovitch functions with the norm

$$
\|f\|_{B_{p}}=\left\{\bar{M}\left[|f(x)|^{p}\right]\right\}^{1 / p}=\left\{\varlimsup_{T \rightarrow \infty} \frac{1}{2 T} \int_{-T}^{T}|f(x)|^{p} d x\right\}^{1 / p}<\infty, 1 \leqslant p<\infty .
$$

The definitions and main properties of the functions in the space $B_{p},(1 \leqslant p<\infty)$, can be found in works [9] or [10].

Assume that the Fourier series of almost periodic in the Besicovitch sense functions is of the form

$$
f(x) \sim \sum_{n=0}^{\infty} A_{n} e^{i \lambda_{n} x}
$$

where

$$
A_{n}=\lim _{T \rightarrow \infty} \frac{1}{2 T} \int_{-T}^{T} f(x) e^{-i \lambda_{n} x} d x
$$

are the Fourier coefficients of functions $f(x) \in B_{p}$, and $\left\{\lambda_{n}\right\},(n=1,2, \ldots)$ are Fourier exponents (or the spectrum of the function).

Some sufficient conditions of absolute Cesáro summability of Fourier series of functions $f(x) \in$ $B_{p},(p \geqslant 1)$, we studied in works [7], [1], [12]. In contrast to periodic functions, in the case of almost periodic functions the required conditions are imposed not only for the smoothness of the functions but also on the behavior of the spectrum of the considered function $\left\{\lambda_{n}\right\}$, $(n=1,2, \ldots)$. This is why we consider two cases: the spectrum of the function (the Fourier exponents) has the only accumulating point at infinity or at zero. In work [7], some tests for the absolute Cesáro summability of Fourier series of functions $f(x) \in B_{2}$ were established, when its spectrum has the only accumulating point at the infinity:

$$
\lambda_{-n}=-\lambda_{n}, \quad\left|\lambda_{n}\right|<\left|\lambda_{n+1}\right| \quad(n=1,2, \ldots) ; \quad \lim _{n \rightarrow \infty}\left|\lambda_{n}\right|=\infty .
$$

The present note is a continuation of work [7] for series of form (1), when the Fourier exponents tend to zero, more precisely,

$$
\lambda_{-n}=-\lambda_{n}, \quad\left|\lambda_{n}\right|<\left|\lambda_{n-1}\right| \quad(n=1,2, \cdots) ; \quad \lim _{n \rightarrow \infty}\left|\lambda_{n}\right|=0 .
$$

At that, as a structural characteristics of the properties of the functions we use the quantity $W_{k}(f ; H)_{B_{2}}$, which is the modulus of average of order $k$ for function $f(x) \in B_{2}$ on $(-\infty, \infty)$ :

$$
W_{k}(f ; H)_{B_{2}}=\sup _{T \geq H}\left\|f_{T^{k}}(x)\right\|_{B_{2}},
$$

where $H>0, k \in \mathbb{N}$,

$$
f_{T^{k}}(x)=(2 T)^{-k} \int_{x-T}^{x+T} d t_{1} \int_{t_{1}-T}^{t_{1}+T} d t_{2} \ldots \int_{t_{k-2}-T}^{t_{k-2}+T} d t_{k-1} \int_{t_{k-1}-T}^{t_{k-1}+T} f\left(t_{k}\right) d t_{k} .
$$


One can check that as $k=1$, the quantity $W_{k}(f ; H)$ possesses all properties similar to the properties of modulus of continuity $\omega_{1}(f ; h)$ :

1. $W(f ; H)$ decreases monotonically as $H \rightarrow+\infty$.

2. If $n$ is a natural number, then

$$
W(f ; n H) \leqslant n W(f ; H),
$$

and $\lambda$ is an arbitrary positive number, then

$$
W(f ; \lambda H) \leqslant(\lambda+1) W(f ; H) .
$$

3. The function $W(f ; H)$ is semi-additive, that is, for all $T_{1}>0, T_{2}>0$,

$$
W\left(f ; T_{1}+T_{2}\right) \leqslant W\left(f ; T_{1}\right)+W\left(f ; T_{2}\right) .
$$

4. The function $W(f ; H)$ is continuous on the interval $0<H<\infty$.

We note that the quantity $W_{k}(f ; H)_{B_{p}}$ was employed earlier in studying the tests for the absolute convergence of the Fourier series of functions $f(x) \in B_{p},(1 \leqslant p<\infty)$ (see, for instance, [13]).

We shall make use of the following auxiliary statements, which were used in work [7].

Lemma 1. If the series

converges, then as $0<\alpha<1$, the series

$$
\sum_{n=0}^{\infty}\left|u_{n}\right|
$$

$$
\sum_{n=1}^{\infty}\left(A_{n}^{\alpha}\right)^{-1} u_{n}
$$

is summable by $|C, \alpha|$ method.

Lemma 2. Assume that a uniformly convergent sequence of measurable integrable on each finite segment functions $\left\{f_{n}(x)\right\}$ is such that

a) $\left\{f_{n}(x)\right\} \in B_{2}, n=1,2, \ldots$;

b) $0 \leqslant f_{1}(x) \leqslant f_{2}(x) \leqslant \ldots \leqslant f_{n}(x) \leqslant \ldots$;

c) $\bar{M}\left\{f_{n}(x)\right\} \leqslant K,(K$ is some constant independent of $n)$.

Then there exists a function $f(x) \in B_{1}$ such that

$$
\lim _{n \rightarrow \infty} f_{n}(x)=f(x), \quad \lim _{n \rightarrow \infty} \bar{M}\left\{f_{n}(x)\right\}=\bar{M}\{f(x)\}
$$

almost everywhere.

Proof. Let $\varepsilon>0$ be an arbitrary number and $N(\varepsilon)$ be such that

$$
\sup _{-\infty<x<\infty}\left|f(x)-f_{N}(x)\right|<\frac{\varepsilon}{3} .
$$

If $\tau$ is the $\frac{\varepsilon}{3}$-almost period of function $f_{N}(x)$, then we have

$$
|f(x+\tau)-f(x)| \leqslant\left|f(x+\tau)-f_{N}(x+\tau)\right|+\left|f_{N}(x+\tau)-f_{N}(x)\right|+\left|f_{N}(x)-f(x)\right|<\varepsilon .
$$

Since the set of $\frac{\varepsilon}{3}$-almost-periods of the function $f_{N}(x)$ is relatively dense and by the last inequality each $\frac{\varepsilon}{3}$-almost period of the function $f_{N}(x)$ is the $\varepsilon$-almost period of the function $f(x)$, it follows that

$$
\lim _{n \rightarrow \infty} f_{n}(x)=f(x) .
$$

The proof of the second part of the lemma is implied immediately by the estimate

$$
\left|\bar{M}\{f(x)\}-\bar{M}\left\{f_{n}(x)\right\}\right| \leqslant \bar{M}\left\{\left|f(x)-f_{n}(x)\right|\right\} \leqslant \sup _{x}\left|f(x)-f_{n}(x)\right| .
$$


By means of Lemma 2 we establish the following statement, which was proved in work [7].

Lemma 3. If we are given a sequence of functions $\left\{\varphi_{n}(x)\right\} \in B_{1},(n=1,2, \cdots)$, and $\varphi_{n}(x) \geqslant 0,(n=1,2, \cdots)$, almost everywhere, then the condition

$$
\sum_{n=1}^{\infty} \bar{M}\left\{\varphi_{n}(x)\right\}<\infty
$$

implies that the series

$$
\sum_{n=1}^{\infty} \varphi_{n}(x)
$$

converges almost everywhere.

\section{MAIN RESULTS}

Here we provide the main results of the paper. First we establish sufficient conditions for $|C, \alpha|$-summability of Fourier series of functions $f(x) \in B_{2}$ for negative values of $\alpha$. Namely, we have the following statement.

Theorem 1. Assume that the spectrum $\Lambda\left\{\lambda_{n}\right\},(n=1,2, \cdots)$, of the function $f(x) \in B_{2}$ satisfies conditions (2) and $\lambda_{n}=O\left(n^{-\delta}\right)(\delta>0)$. If as

$$
0<\beta<2, \quad 0 \leqslant \gamma<1, \quad k>\frac{\gamma+1-\beta / 2}{\beta \delta}, \quad \rho=\frac{\gamma+1-\beta / 2}{\delta}
$$

we have

$$
\sum_{n=1}^{\infty} n^{\rho-1} W_{k}^{\beta}(f ; n)_{B_{2}}<\infty
$$

then the series

$$
\sum_{n=1}^{\infty}\left|A_{n}\right|^{\beta}
$$

is summable by $|C,-\gamma|$ method.

Proof. Let

$$
\sum_{n=0}^{\infty} A_{n} e^{i \lambda_{n} x}
$$

be the Fourier series of a function $f(x) \in B_{2}$. Since

$$
\frac{1}{(2 T)^{k}} \int_{x-T}^{x+T} d t_{1} \int_{t_{1}-T}^{t_{1}+T} d t_{2} \ldots \int_{t_{k-2}-T}^{t_{k-2}+T} d t_{k-1} \int_{t_{k-1}-T}^{t_{k-1}+T} e^{i \lambda_{n} t_{k}} d t_{k}=e^{i \lambda_{n} x}\left\{\frac{\sin \lambda_{n} T}{i \lambda_{n} T}\right\}^{k},
$$

the series

$$
\sum_{n=1}^{\infty} A_{n} e^{i \lambda_{n} x}\left\{\frac{\sin \lambda_{n} T}{i \lambda_{n} T}\right\}^{k}
$$

is the Fourier series for the function $f_{T^{k}}(x)$.

Applying Parseval identity, we obtain

$$
\left\{\sum_{n=1}^{\infty}\left|A_{n}\left\{\frac{\sin \lambda_{n} T}{i \lambda_{n} T}\right\}^{k}\right|^{2}\right\}^{\frac{1}{2}}=\left\|f_{T^{k}}(x)\right\|_{B_{2}} .
$$

Let $\Lambda\left\{\lambda_{n}\right\},(n=1,2, \cdots)$. Then

$$
N_{\nu}=\left\{n: 2^{-\nu} \pi \leqslant \lambda_{n} \leqslant 2^{-(\nu-1)} \pi\right\}
$$


and $m\left(N_{\nu}\right)$ stands for the exponents $\left\{\lambda_{n}\right\}$ in the set $N_{\nu}$ for each fixed $\nu$. Then for $\nu=0$, thanks to Hölder inequality, we have

$$
\sum_{n \in N_{\nu}}\left|A_{n}\right|^{\beta} \leqslant\left\{m\left(N_{\nu}\right)\right\}^{1-\frac{\beta}{2}} \cdot\left\{\sum_{n \in N_{\nu}}\left|A_{n}\right|^{2}\right\}^{\frac{\beta}{2}} .
$$

By the assumption of the theorem we have $\lambda_{n}=O\left(n^{-\alpha}\right)(\alpha>0)$ and therefore,

$$
K_{1} \cdot \frac{1}{2^{\nu+1}} \leqslant \frac{1}{n^{\alpha}} \leqslant K_{2} \cdot \frac{1}{2^{\nu}}
$$

or

$$
K_{1} \leqslant n^{\alpha} \leqslant K_{2} 2^{\nu+1}
$$

where $K_{1}>0, K_{2}>0$ are some constants. Therefore, $m\left(N_{\nu}\right)=O\left\{2^{\nu / \alpha}\right\}$ uniformly in $\nu$.

Since

$$
\frac{\pi}{2^{\nu+1}} \leqslant \lambda_{n} \leqslant \frac{\pi}{2^{\nu}}
$$

it follows that

$$
\frac{\pi T}{2^{\nu+1}} \leqslant \lambda_{n} T \leqslant \frac{\pi T}{2^{\nu}}
$$

Letting $T=2^{\nu-1}$, we obtain that $\lambda_{n} T \leqslant \frac{\pi}{2}$. It yields

$$
\sin \lambda_{n} T \geqslant \frac{2}{\pi} \lambda_{n} T
$$

Hence, by (5) we get the estimate

$$
\left\{\sum_{n \in N_{\nu}}\left|A_{n}\right|^{2}\right\}^{\frac{1}{2}} \preceq W_{k}\left(f ; 2^{\nu}\right)_{B_{2}} .
$$

Since $m\left(N_{\nu}\right)=O\left\{2^{\nu / \alpha}\right\}$, by means of estimate (7) we rewrite inequality (6) as

$$
\sum_{n \in N_{\nu}}\left|A_{n}\right|^{\beta} \preceq 2^{\frac{\nu}{\alpha}\left(1-\frac{\beta}{2}\right)} W_{k}^{\beta}\left(f ; 2^{\nu}\right)_{B_{2}} .
$$

Let $\gamma>0$. By Hölder inequality we have

$$
\begin{aligned}
\sum_{n \in N_{\nu}}\left|A_{n}\right|^{\beta} n^{\gamma} & \leqslant\left\{\sum_{n \in N_{\nu}} n^{2 \cdot \frac{\gamma}{2-\beta}}\right\}^{1-\frac{\beta}{2}} \cdot\left\{\sum_{n \in N_{\nu}}\left|A_{n}\right|^{2}\right\}^{\frac{\beta}{2}} \\
& \preceq 2^{\frac{\nu \gamma}{\alpha}} \cdot 2^{\nu \cdot \frac{1-\beta / 2}{\alpha}} \cdot W_{k}^{\beta}\left(f ; 2^{\nu}\right)_{B_{2}}=2^{\frac{\nu}{\alpha}\left(\gamma+1-\frac{\beta}{2}\right)} \cdot W_{k}^{\beta}\left(f ; 2^{\nu}\right)_{B_{2}} .
\end{aligned}
$$

Summing up this inequality over $\nu$, we find

$$
\sum_{\nu=1}^{\infty} \sum_{n \in N_{\nu}}\left|A_{n}\right|^{\beta} n^{\gamma} \leqslant \sum_{\nu=1}^{\infty} 2^{\frac{\nu}{\alpha}\left(\gamma+1-\frac{\beta}{2}\right)} \cdot W_{k}^{\beta}\left(f ; 2^{\nu}\right)_{B_{2}} .
$$

In view of the monotonicity of $W_{k}(f ; H)_{B_{2}}(H \rightarrow \infty)$ we obtain

$$
\sum_{n=1}^{\infty}\left|A_{n}\right|^{\beta} n^{\gamma} \preceq \sum_{n=1}^{\infty} n^{\frac{\gamma+1-\frac{\beta}{2}}{\alpha}-1} \cdot W_{k}^{\beta}(f ; n)_{B_{2}} .
$$

By condition (4) and Lemma 1, the series $\sum_{n=1}^{\infty}\left|A_{n}\right|^{\beta}$ is $|C,-\gamma|$-summable. The proof is complete. 
Theorem 2. Let the spectrum $\Lambda\left\{\lambda_{n}\right\}(n=1,2, \ldots)$ of a function $f(x) \in B_{2}$ satisfies conditions (2) and $\lambda_{n}=O\left(n^{-\delta}\right)(\delta>0)$. Then as $-1<\alpha<\frac{1}{2}$, the condition

$$
\sum_{\nu=0}^{\infty} 2^{\nu\left(\frac{1}{2}-\alpha\right)} W_{k}\left(f ; \lambda_{2^{\nu}}^{-1}\right)_{B_{2}}<\infty
$$

as $\alpha=\frac{1}{2}$, the condition

$$
\sum_{\nu=0}^{\infty} 2^{-\nu} W_{k}\left(f ; \lambda_{2^{\nu}}^{-1}\right)_{B_{2}}<\infty
$$

as $\alpha>\frac{1}{2}$, the condition

$$
\sum_{\nu=0}^{\infty} 2^{-\nu}\left(\ln 2^{\nu}\right)^{-1 / 2} W_{k}\left(f ; \lambda_{2^{\nu}}^{-1}\right)_{B_{2}}<\infty
$$

imply $|C, \alpha|$-summability of the series

$$
\sum_{n=0}^{\infty} A_{n} \exp \left(i \lambda_{n} x\right)
$$

Proof. We consider the series

$$
\sum_{n=1}^{\infty}\left|\sigma_{n}^{\alpha}(x)-\sigma_{n-1}^{\alpha}(x)\right|=\sum_{n=1}^{\infty}\left(n A_{n}^{\alpha}\right)^{-1}\left|\sum_{k=1}^{n} A_{n-k}^{\alpha-1} k A_{k} \exp \left(i \lambda_{k} x\right)\right|,
$$

where $\sigma_{n}^{\alpha}(x)$ are $n$-th Cesáro means of order $\alpha,(\alpha>-1)$ and $A_{k}$ are the Fourier coefficients of a function $f(x) \in B_{2}$.

According to Lemma 3, to prove the almost everywhere convergence of series (12), it is sufficient to establish the convergence of the series

$$
G(f ; \alpha)=\sum_{n=1}^{\infty} \bar{M}\left\{\left|\sigma_{n}^{\alpha}(x)-\sigma_{n-1}^{\alpha}(x)\right|\right\} .
$$

It is known [7] that

$$
G(f ; \alpha) \leqslant \sum_{\nu=0}^{\infty} 2^{-\nu\left(\alpha+\frac{1}{2}\right)}\left\{\left(\sum_{n=1}^{2^{\nu}-1} \sum_{k=2^{\nu}}^{2^{\nu+1}-1}+\sum_{n=2^{\nu}}^{2^{\nu+1}-1} \sum_{k=n}^{2^{\nu+1}-1}\right) \frac{n^{2} A_{n}^{2}}{(n-k+1)^{2(1-\alpha)}}\right\}^{\frac{1}{2}} .
$$

1. If $-1<\alpha<\frac{1}{2}$, then $2 \alpha<1$ or $2(1-\alpha)>1$. Hence, (see, for instance, [14]),

$$
\sum_{k=2^{\nu}}^{2^{\nu+1}-1}(k-n+1)^{2(\alpha-1)}<K
$$

where $K$ is some constant. Therefore, by relation (14) for $-1<\alpha<\frac{1}{2}$ and inequality (15), we obtain

$$
G(f ; \alpha) \preceq \sum_{\nu=0}^{\infty} 2^{-\nu\left(\alpha+\frac{1}{2}\right)} \sum_{k=0}^{\nu} 2^{k+1}\left\{\sum_{n=2^{k}}^{2^{k+1}-1} A_{n}^{2}\right\}^{\frac{1}{2}} .
$$

Interchanging the order of the summation in the last inequality, we have

$$
G(f ; \alpha) \preceq \sum_{\nu=0}^{\infty} 2^{-\nu\left(\alpha-\frac{1}{2}\right)}\left\{\sum_{n=2^{\nu}}^{2^{\nu+1}-1} A_{n}^{2}\right\}^{\frac{1}{2}}
$$


It follows from estimate (7) that

$$
\sum_{n=2^{\nu}}^{2^{\nu+1}-1} A_{n}^{2} \leqslant W_{k}^{2}\left(f ; \lambda_{2^{\nu}}^{-1}\right)_{B_{2}}
$$

for each $\nu=1,2, \ldots$, or

$$
\left\{\sum_{n=2^{\nu}}^{2^{\nu+1}-1} A_{n}^{2}\right\}^{\frac{1}{2}} \leqslant W_{k}\left(f ; \lambda_{2^{\nu}}^{-1}\right)_{B_{2}}
$$

By inequality $(16)$ and estimate $(17)$ it follows that

$$
\sum_{\nu=0}^{\infty} 2^{-\nu\left(\alpha-\frac{1}{2}\right)}\left\{\sum_{n=2^{\nu}}^{2^{\nu+1}-1} A_{n}^{2}\right\}^{\frac{1}{2}} \preceq \sum_{\nu=0}^{\infty} 2^{\nu\left(\frac{1}{2}-\alpha\right)} W_{k}\left(f ; \lambda_{2^{\nu}}^{-1}\right)_{B_{2}} .
$$

It follows from estimate (9) that series $(13)$ converges almost everywhere. This means that by Lemma 3 series 12 converges almost everywhere. Therefore, as $-1<\alpha<\frac{1}{2}$, series (11) is summable by $|C, \alpha|$ method.

2. Let $\alpha=\frac{1}{2}$. Then by relation (14) we obtain

$$
\begin{aligned}
G(f ; \alpha) & \preceq \sum_{\nu=0}^{\infty} 2^{-\nu\left(\frac{1}{2}+\frac{1}{2}\right)}\left\{\left(\sum_{n=1}^{2^{\nu}-1} \sum_{k=2^{\nu}}^{2^{\nu+1}-1}+\sum_{n=2^{\nu}}^{2^{\nu+1}-1} \sum_{k=n}^{2^{\nu+1}-1}\right) \frac{n^{2} A_{n}^{2}}{n-k+1}\right\}^{\frac{1}{2}}=\sum_{\nu=0}^{\infty} 2^{-\nu}\left\{\sum_{n=1}^{2^{\nu+1}-1} n^{2} A_{n}^{2}\right\}^{\frac{1}{2}} \\
& =\sum_{\nu=0}^{\infty} 2^{-\nu}\left\{\sum_{k=0}^{\nu} \sum_{n=2^{k}}^{2^{k+1}-1} n^{2} A_{n}^{2}\right\}^{\frac{1}{2}} \leqslant \sum_{\nu=0}^{\infty} 2^{-\nu} \sum_{k=0}^{\nu} 2^{k+1}\left\{\sum_{n=2^{k}}^{2^{k+1}-1} A_{n}^{2}\right\} \leqslant \sum_{\nu=0}^{\infty}\left\{\sum_{n=2^{\nu}}^{2^{\nu+1}-1} A_{n}^{2}\right\}^{\frac{1}{2}} .
\end{aligned}
$$

By applying the inequality [15]

$$
\sum_{n=1}^{\infty} d_{n}^{\delta} \leqslant c_{\beta} \sum_{n=1}^{\infty} n^{-\delta}\left(\sum_{\nu=n}^{\infty} d_{\nu}\right)^{\delta}, \quad 0<\delta<1, \quad d_{n} \geqslant 0,
$$

we get

$$
G(f ; \alpha) \preceq \sum_{\nu=0}^{\infty} 2^{-\nu}\left\{\sum_{n=2^{\nu}}^{2^{\nu+1}-1} A_{n}^{2}\right\}^{\frac{1}{2}}
$$

Estimate 17] implies

$$
G(f ; \alpha) \preceq \sum_{\nu=0}^{\infty} 2^{-\nu} W_{k}\left(f ; \lambda_{2^{\nu}}^{-1}\right)_{B_{2}}
$$

and by Lemma 1 and condition $(9)$ this yields the $\left|C ; \frac{1}{2}\right|$-summability of series (11).

3. Assume that $\alpha>\frac{1}{2}$. Then $2-2 \alpha<1$. Thus, interchanging the order of summation and applying of the estimate

$$
\sum_{\nu=2^{m}}^{2^{m+1}-1} \frac{1}{(n-\nu+1)^{2(1-\alpha)}}=O\left(2^{m\left(\alpha+\frac{1}{2}\right)}\right)
$$

by (14) we obtain

$$
G(f ; \alpha) \preceq \sum_{n=0}^{\infty} 2^{-n\left(\alpha+\frac{1}{2}\right)} 2^{n\left(\alpha+\frac{1}{2}\right)}\left\{\sum_{\nu=2^{n}}^{2^{n+1}-1} A_{\nu}^{2}\right\}^{\frac{1}{2}}=\sum_{n=0}^{\infty}\left\{\sum_{\nu=2^{n}}^{2^{n+1}-1} A_{\nu}^{2}\right\}^{\frac{1}{2}}
$$


By applying the inequality [5]

we get

$$
\sum_{n=0}^{\infty}\left(\sum_{k=2^{n}+1}^{2^{n+1}} A_{k}^{2}\right)^{\frac{1}{2}} \leqslant 4 \cdot \sum_{n=2}^{\infty} \frac{\left(\sum_{k=n}^{\infty} A_{k}^{2}\right)^{\frac{1}{2}}}{n(\ln n)^{1 / 2}}
$$

$$
G(f ; \alpha) \preceq \sum_{n=2}^{\infty} n^{-1}(\ln n)^{-\frac{1}{2}}\left(\sum_{\nu=2^{n}}^{2^{n+1}-1} A_{\nu}^{2}\right)^{\frac{1}{2}} \leqslant \sum_{\nu=1}^{\infty} 2^{-\nu}\left(\ln 2^{\nu}\right)^{-\frac{1}{2}} W_{k}\left(f ; \lambda_{2^{\nu}}^{-1}\right)_{B_{2}} .
$$

By condition 10 this implies the almost everywhere convergence of series 13 that by Lemma 1 implies $|C, \alpha|$-summability of series $(11)$ for $\alpha=\frac{1}{2}$. The proof of Theorem 2 is complete.

\section{BIBLIOGRAPHY}

1. L. Leindler. Uber die absolute summierbarkeit der rthogonalreihen // Acta Sci. Math. 22:3-4, 243268 (1961).

2. K. Tandori Uber die orthogonalen funktionen IX. (Absolute summation) // Acta Sci. Math. 21:3-4, 292-299 (1960).

3. M.F. Timan. On absolute convergence and summability of Fourier series // Soobsh. AN Gruz. SSR. 26:6, 641-646 (1961). (in Russian).

4. S.B. Stechkin. On absolute convergence of orthogonal series // Dokl. AN SSSR. 102:2, 37-40 (1955). (in Russian.)

5. L.V. Grepachevskaya. Absolute summability of orthogonal series // Matem. Sborn. 65:3, 370-389 (1964).

6. G. Sunouchi. On the absolute summability of Fourier series // J. Math.Soc. Japan. 1:2, 57-65 (1949).

7. Yu.Kh. Khasanov. On absolute summability of Fourier series of almost periodic functions // Analysis Math. 39:4, 259-270 (2013). (in Russian).

8. S.A. Baron. Introduction to the theory of summability of series. Valgus, Tallin (1977). (in Russian).

9. A. Besicovitch. Almost periodic functions. Cambridge Univ. Press, Cambridge (1932).

10. B.M. Levitan. Almost periodic functions. GITTL, Moscow (1953). (in Russian).

11. M.F. Timan, Yu.Kh. Khasanov. On the absolute summability of Fourier series of almost-periodic Besicovitch functions // Ukrain. Matem. Zhurn. 61:9, 1267-1276 (2009). [Ukrain. Math. J. 61:9, 1499-1510 (2009).]

12. Yu.Kh. Khasanov. On the absolute summability of Fourier series of almost periodic functions // Ukrain. Matem. Zhurn. 65:12, 1716-1722 (2013). [Ukrain. Math. J. 65:12, 1904-1911 (2013).]

13. Yu.Kh. Khasanov. Absolute convergence of Fourier series of almost-periodic functions // Matem. Zametki. 94:5, 745-756 (2013). [Math. Notes. 94:5, 692-702 (2013).]

14. G. Alexits. Convergence problems of orthogonal series. Int. Ser. Mon. Pure Appl. Math. 20. Pergamon Press, New York (1961).

15. G.H. Hardy, J.E. Littlewood, G. Pólya. Inequalities. Cambridge Univ. Press, Cambridge (1934).

Yusufali Khasanovich Khasanov,

Russian-Tajik Slavonic University,

M. Tursunzoda str. 30,

734025, Dushanbe, Republic of Tajikistan

E-mail: yukhas60@mail.ru 